\title{
NORMS ON QUOTIENT SPACES
}

\section{BY ARNOLD LEBOW AND MARTIN SCHECHTER}

Communicated by Bertram Yood, June 23, 1967

1. Perturbation classes. Let $S$ be a subset of a Banach space $a$ over the complex numbers, and assume that $\alpha \delta \subset s$ for each scalar $\alpha \neq 0$. Let $P(\delta)$ denote the set of elements of $a$ that perturb $S$ into itself, i.e., $P(\delta)=\{a \in a: a+s \in \delta$ for all $s \in \delta\}$.

Proposition 1.1. $P(S)$ is a linear subspace of $a$. If $S$ is an open subset of $a$, then $P(\mathcal{S})$ is closed.

PRoposition 1.2. Let $\S_{1} \subset S_{2}$ be two such subsets, and assume that $S_{1}$ is open and $S_{2}$ does not contain any boundary point of $S_{1}$. Then $P\left(S_{2}\right)$ $\subset P\left(s_{1}\right)$.

Proposition 1.3. Assume that $a$ is a Banach algebra with identity e. Let $G$ denote the set of invertible elements in a. If $G s \subset s$, then $P(s)$ is a left ideal. If $\delta G \subset \delta$, then $P(\delta)$ is a right ideal.

Proposition 1.4. $P(G)=R$, the radical of $a$.

Let $G_{l}\left(G_{r}\right)$ denote the set of left (right) invertible elements of $a$, and let $H_{l}\left(H_{r}\right)$ denote the set of elements of $a$ that are not left (right) topological divisors of zero.

Theorem 1.5. $P\left(H_{l}\right) \subset P\left(G_{l}\right)=R=P\left(G_{r}\right) \supset P\left(H_{r}\right)$.

Let $X$ be a Banach space, and let $B(X)[\varkappa(X)]$ denote the set of bounded (compact) linear operators on $X$. Take $a=B(X) / \mathscr{K}(X)$ and let $\pi$ be the canonical homomorphism from $B(X)$ to a. Set

$$
\Phi(X)=\pi^{-1}(G), \quad \Phi_{l}(X)=\pi^{-1}\left(G_{l}\right), \quad \Phi_{r}(X)=\pi^{-1}\left(G_{r}\right) .
$$

It is well known [6] that $\Phi_{l}(X)$ consists of those operators having finite nullity and closed, complemented ranges, and that $\Phi_{r}(X)$ consists of those operators having complemented null spaces and closed ranges with finite codimensions. $\Phi(X)=\Phi_{l}(X) \cap \Phi_{r}(X)$ is the set of Fredholm operators on $X$.

Theorem 1.6. $P(\Phi)=P\left(\Phi_{l}\right)=P\left(\Phi_{r}\right)=\pi^{-1}(R)$.

Let $Z$ be any subset of $\{0, \pm 1, \pm 2, \cdots, \pm \infty\}$, and let $\Phi_{z}$ be the collection of those operators $A \in \Phi_{l}(X) \cup \Phi_{r}(X)$ such that $i(A) \in Z$, where $i(A)=\operatorname{dim} N(A)-\operatorname{dim} N\left(A^{\prime}\right)$.

TheOREM 1.7. $P\left(\Phi_{s}\right)=\pi^{-1}(R)$. 
2. Measures of noncompactness. Let $X, Y$ be Banach spaces, and denote the set of bounded (compact) linear operators from $X$ to $Y$ by $B(X, Y)[\varkappa(X, Y)]$. Let $S_{X}$ denote the unit ball in $X$. For any bounded subset $\Omega$ of $X$ let $q(\Omega)$ denote the greatest lower bound of the numbers $r$ such that $\Omega$ can be covered by a finite collection of spheres of radius $r$. For $A \in B(X, Y)$ set $\|A\|_{q}=q\left[A\left(S_{X}\right)\right]$. Let $\|A\|_{m}$ denote the greatest lower bound of all numbers $\eta$ such that $\|A x\|$ $\leqq \eta\|x\|$ for all $x$ in some subspace having finite codimension. Let $\pi$ denote the canonical homomorphism of $B(X, Y)$ into $B(X, Y) / \mathfrak{K}(X$, $Y)$.

Proposition 2.1. Both $\|\cdot\|_{q}$ and $\|\cdot\|_{m}$ are seminorms and satisfy $\|B A\|_{q} \leqq\|B\|_{q}\|A\|_{q},\|B A\|_{m} \leqq\|B\|_{m}\|A\|_{m},\|A\|_{q} \leqq\|\pi(A)\|,\|A\|_{m}$ $\leqq\|\pi(A)\|,\|A+K\|_{Q}=\|A\|_{q},\|A+K\|_{m}=\|A\|_{m}$ for $K \in \mathcal{K}(X, Y)$.

Theorem 2.2. $\|A\|_{q} / 2 \leqq\|A\|_{m} \leqq 2\|A\|_{\Omega}$.

Definition 2.3. A Banach space $X$ will be said to have the compact approximation property with constant $\gamma$ if for each $\epsilon>0$ and finite set of points $x_{1}, \cdots, x_{n}$ in $X$ there is an operator $K \in \mathcal{K}(X)$ such that $\|I-K\| \leqq \gamma$ and $\left\|x_{j}-K x_{j}\right\|<\epsilon$ for $1 \leqq j \leqq n$.

THEOREM 2.4. If $Y$ has the compact approximation property with constant $\gamma$, then $\|\pi(A)\| \leqq \gamma\|A\|_{q}$. Thus $B(X, Y) / \mathscr{K}(X, Y)$ is complete with respect to the norms induced by $\|\cdot\|_{q}$ and $\|\cdot\|_{m}$.

3. Semi-Fredholm operators. An operator $A \in B(X, Y)$ is in $\Phi_{+}(X, Y)$ if it has finite nullity and closed range.

THEOREM 3.1. An operator $A$ is in $\Phi_{+}(X, Y)$ if and only if for each Banach space $Z$ there is a constant $C$ such that $\|T\|_{m} \leqq C\|A T\|_{m}$, $T \in B(Z, X)$. The constant does not depend on $Z$.

Corollary 3.2. If $A \in \Phi_{+}(X, Y)$ and $X$ has the compact approximation property, then $\|\pi(T)\| \leqq C\|\pi(A T)\|, T \in B(Z, X)$, for any Banach space $Z$.

Definition 3.3. For $A \in B(X, Y)$ set $q_{A}=\mathrm{glb} q[A(\Omega)] / q(\Omega)$, where the glb is taken over all bounded subsets $\Omega$ of $X$.

TheOREM 3.4. $A \in \Phi_{+}(X, Y)$ if and only if $q_{A} \neq 0$.

An operator $A \in B(X, Y)$ is in $\Phi(X, Y)$ if its range is closed and has finite codimension.

TheOREM 3.5. $A \in \Phi_{-}(X, Y)$ if and only if $\beta(A-K)<\infty$ for all $K \in \mathfrak{K}(X, Y)$, where $\beta(E)=\operatorname{codim} \overline{R(E)}$. 
ThEOREM 3.6. $A \in \Phi_{-}(X, Y)$ if and only if for each $Z$ there is a constant $C$ such that $\|T\|_{m} \leqq C\|T A\|_{m}, T \in B(Y, Z)$. The constant $C$ is independent of $Z$.

We now consider the case $X=Y$. Let $r_{\sigma}(A)$ denote the spectral radius of an operator $A$.

THEOREM 3.7. If $\left\|A^{n}\right\|_{m}<1$ for some $n \geqq 1$, then $I-A \in \Phi(X)$ and $i(I-A)=0$.

THEOREM 3.8 .

$$
r_{\sigma}[\pi(A)]=\lim _{n \rightarrow \infty}\left\|A_{n}\right\|_{m}^{1 / n}=\lim _{n \rightarrow \infty}\left\|A^{n}\right\|_{q}^{1 / n}=\max _{\lambda \in \sigma_{\theta}(A)}|\lambda|,
$$

where $\sigma_{e}(A)$ denotes the essential spectrum of $A$ according to any of the usual definitions [8], [9].

CoRollary 3.9. $r_{\sigma}[\pi(A)] \geqq q_{A}$. Hence an operator in $\Phi_{+}(X)$ cannot be a Riesz operator.

Definition 3.10. A space $X$ has the range property if for each $\epsilon>0$ and each $A \in B(X)$ with $\operatorname{dim} N(A)=\infty$ there is a $T \in B(X)$ such that $\|T\|_{q}=1$ and $q\left[T\left(S_{X}\right) \backslash N(A)\right]<\epsilon$. All subprojective [10] spaces have the range property.

THEOREM 3.11. If $X$ has the range property, then $A \in \Phi_{+}(X)$ if and only if $\|T\|_{Q} \leqq C\|A T\|_{a}$ for all $T \in B(X)$.

THEOREM 3.12. If $X$ is subprojective and $\pi(A)$ is not a left zero divisor then $A \in \Phi_{+}(X)$.

CoROLlaRY 3.13. If $X$ is subprojective and has the compact approximation property, then every topological left zero divisor in $B(X) / \mathcal{K}(X)$ is a left zero divisor.

THEOREM 3.14. If $X$ is superprojective [10] and $\pi(A)$ is not a right zero divisor, then $A \in \Phi(X)$.

CoRollary 3.15. If $X$ is both subprojective and superprojective, then every element of $B(X) / \mathcal{K}(X)$ which is not a zero divisor is invertible.

4. Remarks. Some of the results of $\$ 1$ were also obtained by B. Gramsch [12]. The $q$-seminorm was studied by Gol'denšteln, Gokhberg, Markus [1], [2] and Darbo [3]. The basic idea goes back to Kuratowski [11]. For the $q$-seminorm Propostion 2.1 was proved in [1]. The compact approximation property is weaker than the metric approximation property of Grothendieck [4] and is similar to one of Bonsall [5]. 


\section{BIBLIOGRAPHY}

1. L. S. Gol'denštern, I. Ts. Gokhberg and A. S. Markus, Investigation of some properties of bounded linear operators in connection with their q-norms, Uchen. Zap. Kishinevsk. Un-ta 29 (1957), 29-36.

2. L. S. Gol'denšteln and A.S. Markus, On the measure of non-compactness of bounded sets and of linear operators, Studies in Algebra and Math. Anal., pp. 45-54, Izdat. "Karta Moldovenjaskei," Kishinev, 1965. (Russian)

3. G. Darbo, Punti uniti in transformazioni a condiminio non compatto, Rend. Sem. Mat. Padova 24 (1955), 84-92.

4. Alexandre Grothendieck, Produits tensoriels topologiques et espaces nucleaires, Mem. Amer. Math. Soc., no. 16, Amer. Math. Soc., Providence, R. I., 1955.

5. Frank F. Bonsall, Compact linear operators, Lecture notes, Yale University, New Haven, Conn., 1967.

6. Bertram Yood, Properties of linear transformations preserved under addition of a completely continuous transformation, Duke Math. J. 18 (1951), 599-612.

7. Martin Schechter, Riesz operators and Fredholm perturbations, Bull. Amer. Math. Soc. 64 (1968), 1139-1144.

8. K. Gustafson and J. Weidmann, On the essential spectrum, J. Math. Ann. Appl. 25 (1969), 121-127.

9. Martin Schechter, On perturbations of essential spectra, J. London Math. Soc. 1 (1969), 343-347.

10. R. J. Whitley, Strictly singular operators and their conjugates, Trans. Amer. Math. Soc. 113 (1964), 252-261.

11. Casmir Kuratowski, Sur les espaces complets, Fund. Math. 15 (1930), 301-309.

12. B. Gramsch, Über analytische Stroungen und den Index von Fredholm-operatoren auf Banachraumen, University of Maryland, College Park, Md., 1969.

Belfer Graduate School of Science, Yeshiva University, New York, New YORK 10033 